\title{
Jogos Digitais como Estratégia para Desenvolver o Pensamento Computacional nos Anos Finais do Ensino Fundamental
}

\author{
Valéria Maria dos Santos Silva, Juliana Regueira Basto Diniz, Sônia Virgínia \\ Alves França
}

Unidade Acadêmica de Educação a Distância e Tecnologia - Universidade Federal Rural de Pernambuco, Recife - PE, Brasil.

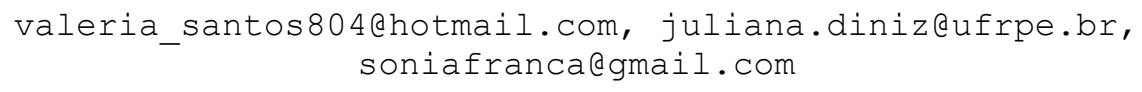

\begin{abstract}
There are a lot of papers relating digital games in classrooms aiming to teach computer science. In some researches, teachers apply digital games in their classes to develop computational thinking among students. This paper presents a survey that relates papers published between 2014 to 2018. Analyzing the results, we identified some advantages and disadvantages in using digital games in education. Some activities were selected to apply in a school in countryside of Pernambuco, state in Brazil. The results of the first stage of this research are presented in this paper in a experiment with 25 students of 9 th year.
\end{abstract}

Resumo. É possível encontrar vários trabalhos que relatam o uso de jogos digitais em sala de aula com o objetivo de ensinar conceitos de ciência da computação. $\mathrm{Na}$ realização de algumas pesquisas os professores aplicam os jogos digitais em sala de aula com o objetivo de desenvolver o pensamento computacional nos alunos. Este artigo apresenta uma revisão sistemática de literatura, que teve como objetivo identificar artigos publicados entre o periodo de 2014-2018. Na análise dos resultados, foram identificadas vantagens e desvantagens na utilização dos jogos, bem como foram elencadas um conjunto de atividades para aplicação de pesquisa de campo numa escola, da zona da Mata do estado de Pernambuco. Os resultados da primeira etapa da pesquisa de campo também são apresentados como achados da pesquisa através de um experimento com 25 alunos do $9^{\circ}$ ano do Ensino Fundamental II.

\section{Introdução}

Levando em consideração os avanços tecnológicos, a Sociedade Brasileira de Computação (SBC) incentiva ações que permitam o ensino dos conceitos da Computação a partir do ensino básico. $\mathrm{O}$ estímulo por parte do órgão ocorre com a intenção de que desde cedo, os princípios e habilidades sejam trabalhados nos estudantes, e desse modo contribuir para a prática da lógica e resolução de problemas, assim como, estimular o interesse pela área, aumentando o número de profissionais no país. Uma das formas de introduzir os conceitos de informática nas escolas é através dos jogos digitais. Marinho e colegas relatam que muitos estudos defendem não apenas o uso dos jogos nas escolas, mas também que o desenvolvimento de jogos digitais por alunos como estratégias de aprendizagem [Marinho et al. 2011]. Os jogos são considerados importantes para o desenvolvimento da coordenação motora e aprendizagem intelectual. Os jogos trabalham aspectos 
cognitivos [Ramos, 2013], sendo esta uma forma de abrir a mente para a criatividade e aprendizado.

Outro fator relevante para o uso dos jogos é o desenvolvimento do pensamento computacional. O Pensamento Computacional (PC) é definido por Wing (2006), como um processo de resolução de problemas com práticas fundamentadas na Ciência da Computação capaz de estimular o desenvolvimento de habilidades como decomposição, abstração, generalização, raciocínio algorítmico e modularização. Para Wing, o PC é um pensamento do ser humano, ao invés de um pensamento mecânico ou de máquina. Ao fazer uso do PC, o indivíduo desenvolve habilidades importantes, o que pode lhe proporcionar, em curto prazo, sucesso em sua vida pessoal e profissional.

O presente artigo trata-se de uma Revisão Sistemática da Literatura (RSL) com o objetivo de analisar os artigos sobre o uso de jogos digitais para o ensino de programação e desenvolvimento do Pensamento Computacional. A pesquisa foi realizada em trabalhos publicados sobre o tema nos últimos cinco anos (2014 a 2018). A busca foi feita em bases de pesquisa dos Anais de Simpósio Brasileiro de Informática na Educação (SBIE), Anais do Workshop de Informática na Escola (WIE) e nos Anais dos Workshops do Congresso Brasileiro de Informática na Educação (WCBIE). Além disso, apresenta como resultado, uma proposta transversal para o ensino do pensamento computacional e programação utilizando jogos.

O artigo encontra-se organizado da seguinte maneira: a Seção 2 apresenta estudos que tratam do ensino de computação. A Seção 3 descreve a metodologia utilizada na execução da pesquisa. A Seção 4 descreve como foi feita a seleção dos trabalhos utilizados para a produção desta pesquisa, como também apresenta a lista de artigos pesquisados. A Seção 5 expõe os resultados obtidos a partir das respostas das questões de pesquisa levantadas na metodologia. A Seção 6 apresenta uma abordagem proposta para a aplicação nos anos finais do ensino fundamental e uma lista com as atividades propostas. A Seção 7 traz os resultados preliminares da experimentação dessa proposta numa escola no interior de Pernambuco. Por fim, as considerações finais sobre o referido trabalho são apresentadas na seção 8 , sendo seguida pelas referências bibliográficas.

\section{Referencial Teórico}

O Model Curriculum for K-12 Computer Science (SEEHORN et al. 2011) apresenta a maneira como a Computação como Ciência pode contribuir, de maneira interdisciplinar, na busca de soluções de problemas. Na economia americana, a ciência da computação é peça fundamental, mas, no entanto, apenas 35 estados americanos permitem aos estudantes cursarem cursos de computação no ensino médio.

Nos Estados Unidos, algumas instituições, tais como, Association for ComputingMachinery, Code.org, Computer Science TeachersAssociation, Cyber Innovation Center e NationalMath e Science Initiative tem trabalhado conjuntamente na definição e desenvolvimento de um padrão conceitual para o ensino da computação na educação básica (Computer Science Education). O denominado K-12 Computer Science Framework1 apresenta-se como uma proposta para o sistema educacional americano se adaptar a visão dos estudantes do século 21 que não são apenas usuários de computador, mas também criadores que são proficientes nos conceitos e práticas da ciência da computação (CSTA et al. 2010).

1https://k12cs.org/framework-statements-by-grade-band/ 
De acordo com o modelo proposto, no ensino fundamental I, o estudante aprende como passar instruções ao computador através de uma sequência de ações como peças de um quebracabeça, criando algoritmos computacionais como desenhos. Pela pouca idade, o estudante entende a computação como uma experiência criativa e com uma ferramenta para sua expressão pessoal. Esse mesmo estudante ao chegar ao ensino fundamental II irá evoluir no uso dos conceitos e compreensão de como o computador trabalha, uma vez que usa o computador e os princípios computacionais para aprimorar suas experiências de aprendizagem em outras disciplinas. A computação apresenta-se como um instrumento para representar e resolver problemas. Quando chega ao ensino médio esse estudante enxerga oportunidades dentro de sua comunidade para aplicar computação de maneira inovadora. Os conceitos e práticas da ciência da computação podem ser empregados para efetivar mudanças autênticas em baixa ou larga escala de interesses.

No Brasil, a Sociedade Brasileira de Computação elaborou uma proposta de diretrizes para o ensino de computação nos ensinos fundamental e médio entregando-a em julho de 2018 ao Conselho Nacional de Educação. Este documento contempla aspectos relativos à que conceitos devem ser trabalhados em cada ano da educação básica.

Nos Anos Iniciais do ensino fundamental, por exemplo, devem ser trabalhados conceitos relacionados às estruturas abstratas necessárias à resolução de problemas no eixo de Pensamento Computacional. Espera-se que os estudantes entendam as noções básicas de algoritmo e consigam elaborá-los para solucionar diferentes problemas através de linguagens imagéticas ou mesmo a sua própria linguagem natural. Dessa forma, sugere-se que o Pensamento Computacional seja trabalhado inicialmente de forma desplugada nos Anos Iniciais. Para os anos finais do ensino fundamental, os estudantes devem dominar as principais técnicas para construir soluções algorítmicas de maneira a serem executadas por máquinas e/ou computadores, podendo analisar de maneira crítica os problemas e suas possíveis soluções.

\section{Metodologia}

A abordagem utilizada na pesquisa é qualitativa a fim de poder criar argumentações para o seu desenvolvimento. $\mathrm{O}$ objetivo da revisão sistemática foi pesquisar e selecionar artigos que apresentam e discutem práticas aplicadas em sala de aula para promover o desenvolvimento do pensamento computacional através de jogos digitais. A metodologia da pesquisa é descritiva visando ampliar o conhecimento sobre jogos digitais e como eles podem ser utilizados para ensino de programação. Após a realização da pesquisa bibliográfica, foi selecionado um conjunto de atividades a serem aplicadas nas escolas da rede pública da região de Surubim, na região da Zona da Mata Norte do estado de Pernambuco.

\subsection{Questões de pesquisa}

Esta revisão sistemática de literatura tem por objetivo apresentar informações relevantes sobre o contexto do ensino de programação através do uso de jogos digitais. Desse modo foram elaboradas Questões de Pesquisa (QP), com a finalidade de extrair dos dados obtidos, informações relevantes para a construção do trabalho. Seguem as QP:

- QP1: Quais conceitos/conhecimentos de programação são abordados/ensinados, com a aplicação do jogo?

- QP2: Quais as práticas pedagógicas mais utilizadas como metodologia, para aplicar o estudo?

- QP3: Quais métodos de avaliação da aprendizagem mais utilizados?

- QP4: Quais vantagens e/ou limitações foram encontradas para aplicação da metodologia? 


\subsection{Condução da busca}

Para realizar esse trabalho foi feita uma pesquisa nos principais meios científicos brasileiros relacionados à informática na educação, conforme pode ser visto na Tabela 1. Foi realizada uma busca manual, nas bases de pesquisas brasileiras, (SBIE), (WCBIE) e na (WIE), através da expressão norteadora "jogos digitais". A busca foi refinada através dos critérios "apenas artigos", "pesquisas publicadas de 2014 a 2018" nos idiomas "português" e "inglês".

\section{Tabela 1. Fontes de pesquisa de publicações nacionais}

\begin{tabular}{|l|l|}
\hline \multicolumn{1}{|c|}{ Sigla } & \multicolumn{1}{c|}{ Fonte } \\
\hline SBIE & Simpósio Brasileiro de Informática na Educação \\
\hline WCBIE & Workshops do Congresso Brasileiro de Informática na Educação \\
\hline WIE & Workshop de Informática na Escola \\
\hline
\end{tabular}

Para o artigo ter sido selecionado, ele deveria ter em seu título a expressão de pesquisa. Para uma análise mais profunda foi lido o resumo dos artigos, de modo a escolher os que atendessem os critérios de inclusão, como mostra a Tabela 2. Em relação aos critérios de exclusão, foram desconsiderados os trabalhos que não trariam contribuições à pesquisa, e também estão ilustrados na Tabela 2. Artigos que apresentavam uma revisão sistemática de literatura não foram selecionados, sendo considerados apenas aqueles que descrevem ou aplicam alguma prática pedagógica ou descrevem o uso de jogos digitais como ferramentas de ensino de programação.

Tabela 2. Critérios de inclusão e exclusão

\begin{tabular}{|c|c|}
\hline Critérios de Inclusão (CI’s) & Critérios de Exclusão (CE's) \\
\hline $\begin{array}{l}\text { CI1. Artigos publicados entre os anos de } 2014 \text { e } \\
\text { 2018. } \\
\text { CI2. Trabalhos escritos em português ou inglês. } \\
\text { CI3. Artigos completos (com quatro páginas ou } \\
\text { mais). } \\
\text { CI4. Publicações que possuam em seu título a } \\
\text { expressão "jogos digitais AND ensino de } \\
\text { programação". } \\
\text { CI5. Artigos que apresentam e discutem o uso de } \\
\text { jogos digitais para o ensino de programação no } \\
\text { ensino fundamental. }\end{array}$ & $\begin{array}{l}\text { CE1. Artigos no formato de revisão sistemática de } \\
\text { literatura. } \\
\text { CE2. Artigos que não possuíam em seu título a } \\
\text { expressão norteadora de pesquisa. } \\
\text { CE3. Artigo duplicado. } \\
\text { CE4. Artigos voltados para o ensino de } \\
\text { programação no ensino superior ou na educação } \\
\text { infantil. }\end{array}$ \\
\hline
\end{tabular}

\section{Seleção de Trabalhos}

Aplicando a expressão de busca foram selecionados 97 artigos, cujos resultados podem ser vistos na Tabela 3.

Tabela 3. Seleção de trabalhos

\begin{tabular}{|l|r|r|r|}
\hline Fonte de Pesquisa & Total & CI's & CE's \\
\hline SBIE & 39 & 07 & 32 \\
\hline WCBIE & 29 & 04 & 25 \\
\hline WIE & 29 & 06 & 23 \\
\hline Total & 97 & 17 & 80 \\
\hline
\end{tabular}

Após a leitura do resumo dos artigos e aplicado os critérios de inclusão foram selecionados 17 artigos, ao aplicar os critérios de exclusão, 76 artigos foram excluídos. Os artigos descartados estavam relacionados à pesquisa bibliográfica de revisão sistemática, relatos de experiência com jogos no ensino médio, infantil ou superior. Os artigos selecionados foram lidos para responder as questões de pesquisa. A listagem completa dos 17 artigos está disponível na tabela 4. 
Tabela 4. Lista de Artigos Selecionados

\begin{tabular}{|c|c|c|c|}
\hline ID & Título & Evento & Ano \\
\hline$\overline{\mathrm{E} 01}$ & $\begin{array}{l}\text { Jogos digitais educacionais como instrumento didático no processo de } \\
\text { ensino-aprendizagem das operações básicas de matemática. }\end{array}$ & SBIE & 2014 \\
\hline E02 & $\begin{array}{l}\text { Um framework para criação de jogos voltados para o ensino de lógica } \\
\text { de programação. }\end{array}$ & SBIE & 2014 \\
\hline E03 & $\begin{array}{l}\text { Conquistando com o Resto: Virtualização de um Jogo para o Ensino de } \\
\text { Matemática. }\end{array}$ & SBIE & 2014 \\
\hline E04 & $\begin{array}{l}\text { Desafios e oportunidades ao ensino do pensamento computacional na } \\
\text { educação básica no Brasil. }\end{array}$ & WCBIE & 2015 \\
\hline E05 & $\begin{array}{l}\text { Um relato de experiência da aplicação de videoaulas de programação } \\
\text { de jogos digitais para alunos da educação básica. }\end{array}$ & WIE & 2016 \\
\hline E06 & $\begin{array}{l}\text { Jogos Digitais no desenvolvimento de conceitos matemáticos sob } \\
\text { perspectiva BYOD e abordagem m-Learning na escola pública. }\end{array}$ & WIE & 2016 \\
\hline E07 & $\begin{array}{l}\text { Experiência de Uso de Jogos Educacionais Digitais } \\
\text { Individuais em Contextos de Colaboração. }\end{array}$ & WIE & 2016 \\
\hline E08 & $\begin{array}{l}\text { FORCA: Um editor de jogo educativo personalizável exportável como } \\
\text { objeto de aprendizagem. }\end{array}$ & SBIE & 2017 \\
\hline E09 & $\begin{array}{l}\text { A Utilização de Role Playing Games Digitais como Ferramenta } \\
\text { Complementar no Processo de Aprendizagem de Crianças Deficientes } \\
\text { Visuais. }\end{array}$ & SBIE & 2017 \\
\hline E10 & $\begin{array}{l}\text { Mensurando o desenvolvimento do Pensamento Computacional por } \\
\text { meio de mapas auto-organizáveis: um estudo preliminar em uma } \\
\text { Oficina de Jogos Digitais. }\end{array}$ & WCBIE & 2017 \\
\hline E11 & $\begin{array}{l}\text { Desenvolvimento de games na Língua Inglesa: lógica e criatividade na } \\
\text { construção do conhecimento. }\end{array}$ & WCBIE & 2017 \\
\hline E12 & $\begin{array}{l}\text { A Experiência de Alunos de uma Escola Indígena nos } \\
\text { Primeiros Contatos com Jogos Digitais de Matemática. }\end{array}$ & WIE & 2017 \\
\hline E13 & $\begin{array}{l}\text { Aprendizagem Baseada em Projetos: Relato de Introdução da Lógica } \\
\text { no Ensino Fundamental }\end{array}$ & WIE & 2017 \\
\hline$\overline{\text { E14 }}$ & $\begin{array}{l}\text { Uma Abordagem para o Desenvolvimento de Jogos Digitais } \\
\text { Educativos no Ensino Básico. }\end{array}$ & SBIE & 2018 \\
\hline E15 & $\begin{array}{l}\text { Desenvolvimento e avaliação de um jogo digital educacional sobre } \\
\text { aspectos socioculturais de países hispano falantes. }\end{array}$ & SBIE & 2018 \\
\hline E16 & $\begin{array}{l}\text { O circuito quatro desafios: Atividade lúdica apoiada pelo Pensamento } \\
\text { Computacional. }\end{array}$ & WCBIE & 2018 \\
\hline E17 & $\begin{array}{l}\text { Jogos Educativos no Ensino de Matemática: Qual a Melhor } \\
\text { Abordagem? }\end{array}$ & WIE & 2018 \\
\hline
\end{tabular}

A Figura 1 mostra a quantidade de artigos publicados sobre jogos digitais no ensino de programação, distribuídos por ano e evento.

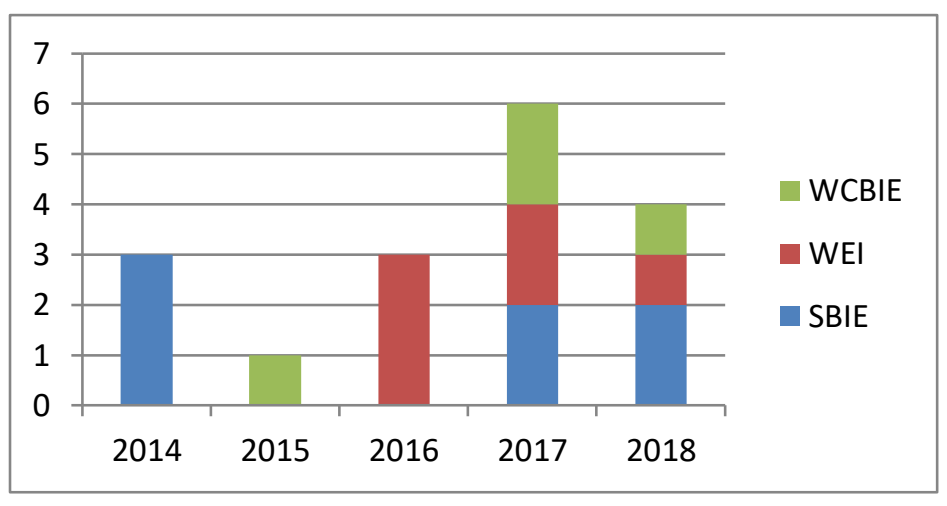

Figura 1. Número de artigos publicados entre 2014 e 2018

De acordo com o gráfico na Figura 1 é possível observar, o crescimento do número de publicações que abordam o uso de jogos digitais para o ensino de programação entre os anos de 
2014 e 2017, com uma queda no ano de 2015 e o mesmo se repete no ano de 2018, considerando todos os eventos.

\section{Resultados da Pesquisa Bibliográfica}

A presente seção expõe os resultados obtidos a partir das respostas das questões de pesquisa (QP) levantadas nesta RSL. A identificação dos estudos é representada pela coluna ID, conforme descrito na Tabela 4. Os resultados serão apresentados e discutidos de forma detalhada, seguindo as questões de pesquisa apresentados na Seção 2.1.

QP1: Quais conceitos/conhecimentos de programação são abordados/ensinados, com a aplicação do jogo?

Os estudos [E2, E4, E11, E13 e E16] abordam os conceitos de lógica de programação. Os estudos [E5 e E13] ensinam comandos e instruções de ensino de programação. Os estudos [E8 e E14] abordam um editor para elaboração de um jogo educativo personalizável como objeto de aprendizagem. $\mathrm{O}$ [E10] analisa programas desenvolvidos em Scratch por meio de mapas autoorganizáveis.

\section{QP2: Quais as práticas pedagógicas mais utilizadas como metodologia, para aplicar o estudo?}

O uso de jogos digitais foi aplicado em 76,47\% dos estudos, o que representa 13 artigos [E1, E3, E4, E5, E6, E7, E8, E9, E11, E12, E14, E15, E16]. Nos estudos [E13 e E17], utilizou-se o método de computação desplugada, como também de jogos digitais, e nos estudos [E2 e E10] foram aplicados as práticas de linguagem de programação.

\section{QP3: Quais métodos de avaliação da aprendizagem mais utilizados?}

Os trabalhos [E1, E4 E6, E15 e E17] avaliam a aplicação do jogo por meio de questionários e observação visual. $\mathrm{O}$ estudo [E5] aplicou um questionário on-line e observação visual. $\mathrm{O}$ estudo [E7] realizou uma análise individual e análise em grupo através de teste de conhecimento e questionário. O trabalho [E10] realizou uma análise observacional. Os estudos [E2, E8, E9, E11 e E12] não especificaram. No estudo [E13] o projeto estava em andamento.

\section{QP4: Quais vantagens e/ou limitações foram encontradas para aplicação da metodologia?}

Vantagens: Os estudos [E1, E9, E12, E16 e E17] apresentam como vantagem o trabalho em equipe, motivação ao praticar as atividades. [E3] facilita na aprendizagem de conceitos fundamentais de operação de divisão. $\mathrm{O}$ trabalho [E5] facilita a compreensão de programação de jogos, motivou a aprender outros conceitos de programação. O trabalho [E6] desenvolve habilidades de raciocínio logico-matemático, resolução de problemas. $\mathrm{O}$ estudo [E7] possibilita a aquisição de novos conhecimentos e o desenvolvimento de habilidades cognitivas e metacognitiva. O trabalho desenvolvido no estudo [E8, E11] motivou os alunos e aumentou o interesse pelos conteúdos abordados em sala de aula. No estudo [E13] observou-se que os alunos apresentavam autonomia na construção dos conhecimentos e evolução do raciocínio logico. No estudo [E15] observou-se o desenvolvimento de competências intercultural. Os estudos [E4 e E10] não especificaram vantagens.

Limitações: Em 70,58\% (12) dos estudos [E1, E2, E3, E4, E5, E7, E9, E10, E11, E13, E14 e E15] não especificaram suas limitações. O estudo [E6] apresentou como limitação, infraestrutura tecnológica precária e ausência de formação de professores. No estudo [E8] observou-se falta de aprofundamento sobre a elaboração de editor de jogos educativos. O estudo [E12] os alunos apresentam poucos conhecimentos em informática, dificuldades em utilizar o mouse e o teclado. 
No estudo [E16] os alunos apresentam falta de conhecimento no assunto abordado, incompatibilidade de metodologia, individualidade e dificuldade de ouvir sugestões por parte dos alunos. No estudo [E17], o jogo foi aplicado na plataforma digital e também sem recursos tecnológicos, sendo que a forma desplugada não motivou os alunos, pois esses apresentavam dificuldades em executar os comandos solicitados.

\section{Proposta Transversal de Ensino}

A presente revisão sistemática de literatura é complementada pela pesquisa de campo em escola de educação básica, na zona da mata norte de Pernambuco, com o intuito de observar como as questões referentes ao ensino do pensamento computacional e da programação podem ser incorporadas ao cotidiano da escola de forma transversal nas disciplinas e através de atividades específicas que envolvam jogos digitais. Assim, apresenta-se aqui uma proposta transversal para ensino do pensamento computacional e programação utilizando jogos. Inicia-se pela computação desplugada, pois através dela é possível desenvolver a linha de raciocínio lógico, sem a necessidade de recursos computacionais. A técnica classificada como computação desplugada foi desenvolvida por Bell e colegas (2011) e consiste em ensinar os fundamentos da computação, através de atividades, sem o uso do computador (BELL et al. 2011). Tais atividades têm despertado o interesse de professores e pesquisadores, e tem sido empregada em diversos países ao redor do mundo (SCAICO et al. 2012).

Isso é importante no contexto do presente trabalho, pois diversas escolas da rede de educação pública brasileira estão desatualizadas quando se falam em recursos computacionais, viabilizando que o referido trabalho possa ser aplicado na região. Para a escolha das atividades, foram utilizadas como referência, as atividades propostas por Bell, no livro Computer Science Unplugged (BELL et al. 2011) e são descritas a seguir: 1)Números Binários; 2)Representação de Imagem; 3) Programação em blocos; 4) Jogo digital Lightbot.

A primeira atividade é simples e objetiva, ensina como ocorre o armazenamento dos dados nos computadores, através de uma série de zeros e uns (sistema binário) com apenas esses dois dígitos os dados são transmitidos e apresentados para o usuário como palavras e números. Em seguida, parte-se para a atividade que utiliza a representação de uma imagem utilizando zeros e uns. A seguir, parte-se para uma ação de maior complexidade, ensinando noções de programação em blocos, culminando com o lightbot que é um jogo online, tendo um robô como personagem principal, cuja função é se movimentar para acender as luzes dos ladrilhos de cor escura. $\mathrm{O}$ jogador deve executar uma série de comandos (andar, acender, girar para esquerda ou para a direita e pular). Os comandos devem ser organizados de forma que, quando executados em sequência, façam o robô atingir o objetivo esperado. Após o término das etapas, espera-se que estimular o desenvolvimento do raciocínio lógico, pensamento computacional, noções básicas de programação, a partir de comandos visuais e jogos digitais e consequentemente trabalhar a capacidade de resolver problemas.

\section{Aplicação das Atividades na Escola}

Como esse projeto está em andamento, até o momento da construção deste artigo, foram aplicadas as duas primeiras atividades, numa turma do $9^{\circ}$ ano, (Ensino Fundamental II) da Escola Municipal São José, escola pública da Zona da Mata Norte, do estado de Pernambuco. Os estudantes desta turma realizam uma vez na semana, no período vespertino, uma aula de reforço. A turma é composta por uma média de 30 alunos. O professor de matemática da turma acompanhou a aplicação das atividades e o processo de aprendizagem. Os materiais utilizados para a realização das atividades consistiram em papel e lápis. Para um melhor aproveitamento das atividades e para que os alunos pudessem entender e tirar suas dúvidas a cerca da resolução das 
questões, foi aplicado uma atividade por aula. Foram realizados três encontros, com duração de 1 hora cada.

Com o objetivo de avaliar o nível de conhecimento dos alunos em relação aos conceitos de ciência da computação, e o interesse que os mesmo possuíam em assuntos relacionados à área, foi aplicado um questionário contendo 3 questões, antes do início das atividades (pré-teste), e após o término de todas as atividades (pós-teste). São elas: a) você já utilizou o computador para realizar alguma atividade escolar, ou outras atividades do seu interesse? Sim() Não(), b) O que você compreende por pensamento computacional? b) O que você acha que significa computação desplugada? Os conceitos computacionais trabalhados nas atividades foram: sequência e padrão sequencial, criptografia, representação de imagens, busca e ordenação. Inicialmente foi apresentada aos alunos a finalidade das atividades e em seguida, lhes foi solicitado que respondessem o pré-teste. Os alunos deveriam responder as questões com base em seus conhecimentos prévios ou fazendo uso do seu entendimento pessoal. Nos resultados obtidos com as respostas dos alunos no pré-teste, observou-se que mesmo sem aulas prévias sobre computação, eles possuíam algum conhecimento sobre o assunto abordado. Ao mesmo tempo também foram analisadas as respostas desses mesmos alunos no pós-teste, com o objetivo de fazer comparações entre elas. Na figura 2, encontram-se as respostas de um mesmo aluno no préteste e pós-teste. Nos dois casos as respostas do aluno estão semelhantes à definição de Computação Desplugada e Pensamento Computacional.
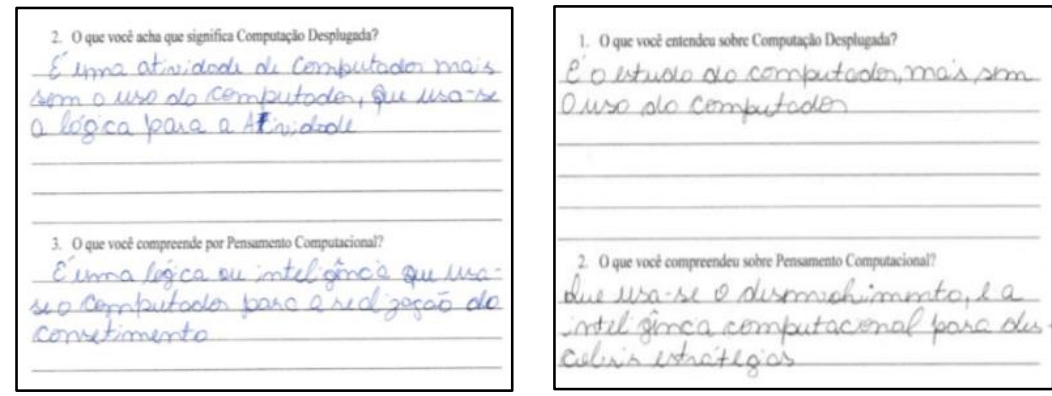

Figura 2 - Resposta de um mesmo aluno no pré-teste e pós-teste

Em alguns casos específicos, os alunos não sabiam ou não tinha ouvido falar em computação desplugada e pensamento computacional, entretanto após a participação nas atividades os alunos conseguiram entender os conceitos de cada um. Na figura 3, encontram-se as respostas de um aluno na questão sobre computação desplugada no pré-teste e ao lado a sua resposta no pós-teste. $\mathrm{Na}$ figura 3 , observa-se que o aluno tenta explicar que é computação desplugada no pré-teste, mas relacionando a definição ao uso de rede social e acesso a internet. Já na resposta do pós-teste o aluno relaciona a atividades do computador feitas sem o uso do mesmo.

$\mathrm{Na}$ atividade Representação de Imagens ocorreu um problema com o transporte escolar e apenas 9 alunos compareceram. Nesta atividade, os alunos seguiram um passo a passo para chegar a um desenho específico. A grade onde o desenho foi feito representava os pixels da tela do computador. A folha de atividade continha três grades onde cada uma "escondia" um desenho diferente. Esse desenho era obtido na medida em que os pixels eram preenchidos, seguido um código de decodificação. Esta folha de atividade foi retirada do livro, Computer Science Unplugged, produzido por Tim Bell, Ian H. Witten e Mike Fellows (2011). 

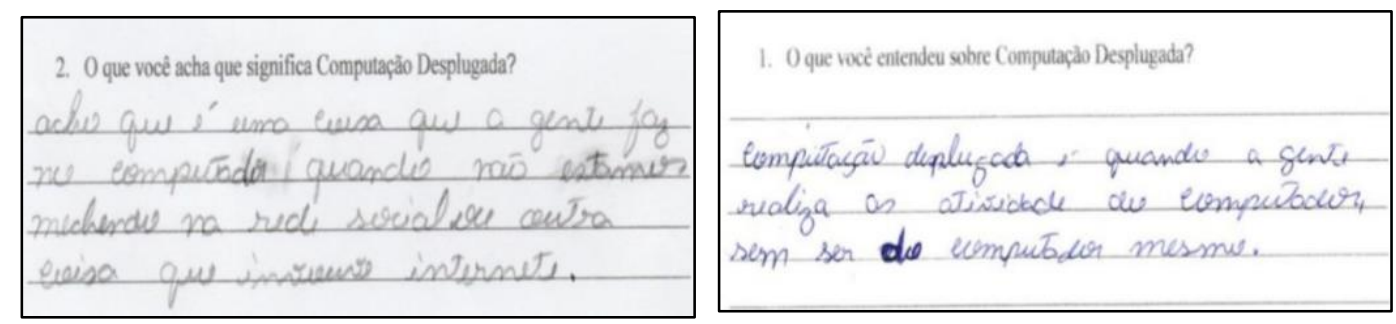

Figura 3. Resposta de um aluno no pré-teste e pós-teste sobre computação desplugada.

Também foi solicitado aos alunos que descrevessem o nível de dificuldade que encontraram em cada um dos desenhos da atividade em: fácil, médio, difícil. A maioria dos alunos classificaram os desenhos como fácil. $\mathrm{Na}$ Tabela 5, encontra-se um comparativo entre os desenhos da atividade 2 e o nível de dificuldade encontrada pelos alunos, para cada um dos desenhos. Outros fatores observados referem-se à falta de erros no preenchimento dos pixels. Alguns alunos sentiram dificuldade em preencher os pixels quando esse iniciava por zero, já que nesse caso a linha começa com um pixel preto.

Tabela 5. Representação do nível de dificuldade dos desenhos da atividade 2

\begin{tabular}{|l|c|c|c|}
\hline & Fácil & Médio & Difícil \\
\hline Desenho 1 & 8 & 1 & 0 \\
\hline Desenho 2 & 1 & 5 & 3 \\
\hline Desenho 3 & 3 & 2 & 4 \\
\hline
\end{tabular}

\section{Conclusões}

Nos resultados obtidos através da pesquisa bibliográfica, foi possível identificar, de acordo com os relatos, uma melhora significativa na aprendizagem dos alunos, com uso dos jogos na sala de aula. Os alunos permaneceram motivados e desenvolveram as atividades proposta com êxito. Entre as dificuldades e limitações encontradas pelos pesquisadores na aplicação do jogo, se encontram a falta de infraestrutura nas escolas e a falta de qualificação dos professores com o uso dos jogos. A partir das análises dos artigos foi possível identificar que, para obter resultados positivos, é preciso aplicar os jogos corretamente. Uma vez que os jogos podem ser classificados como ferramentas para desenvolver o aprendizado do aluno de forma lúdica, esses devem ser educativos e bem estruturados, pois devem auxiliar o professor no processo de ensino aprendizagem, mas não substituir totalmente a presença de um docente na sala de aula. Observa-se que a aplicação de atividades utilizando o método da computação desplugada, também pode ser considerada lúdica e atrativa, assim como a utilização dos jogos digitais, pois além de permitir apresentar o conteúdo, também chamou a atenção dos alunos, características encontradas com a aplicação dos jogos digitais para o ensino de programação. Como trabalho futuro, pretende-se dar continuidade a aplicação das atividades 3 e 4, na escola e a partir dos resultados obtidos, propor um plano de ação para incorporar tais práticas e estratégias que envolvam o uso de jogos digitais, adaptados à realidade brasileira, sobretudo a regiões interioranas da região nordeste. Essa metodologia confrontará resultados obtidos na revisão de sistemática de literatura e os dados observados na pesquisa de campo, contemplando as séries finais do ensino fundamental brasileiro.

\section{Agradecimentos}

Os autores agradecem o apoio da Pró-reitoria de Pesquisa e Pós-graduação da Universidade Federal Rural de Pernambuco no desenvolvimento deste trabalho, através do incentivo à pesquisa, e ao CNPQ, através da concessão de bolsa de iniciação científica. 


\section{Referências}

Bell, T.; Witten, I. e Fellows, M. (2011).“Computer Science Unplugged - Ensinando Ciência da Computação sem o uso do Computador". Disponível em: $<$ http://csunplugged.org/>. Acesso em janeiro de 2019.

Brackmann, C. P. (2017). "Desenvolvimento do pensamento computacional através de atividades desplugadas na educação básica". Disponível em: $<$ https://www.lume.ufrgs.br/handle/10183/172208 > . Acesso em: fevereiro de 2019.

Marinho, A. R. da S.; Morais, P. S. de M.; Souza, G. R. de; Nascimento, A. S. L. do. "Relato de Experiência Vivenciada no PIBID sobre a Utilização da Computação Desplugada, a Hora do Código e do Scratch no Ensino Médio". Disponível em: $<$ http://portaldeconteudo.sbc.org.br/index.php/wei/article/view/3507/3466>. Acesso em: Janeiro de 2019.

Melo, L. de A.; Costa, T. K. de L.; Batista, A. C. D. (2013). "Pense bem: proposta e desenvolvimento de jogo digital para ensino de computação na educação básica". Disponível em: <http://www.br-ie.org/pub/index.php/sbie/article/view/2513/2171>. Acesso em: Fevereiro de 2019.

Ramos, D. K., 2013. Jogos cognitivos eletrônicos: contribuições à aprendizagem no contexto escolar. Ciências \& Cognição (UFRJ). Disponível em: $<$ http://pepsic.bvsalud.org/scielo.php?script=sci_arttext\&pid=S180658212013000100002>. Acesso em: Março de 2019.

Rodrigues, S. R. C.; Eduardo Aranha, E.; Silva, T. R. da. (2018). "Computação Desplugada no Ensino de Programação: Uma Revisão Sistemática da Literatura". Disponível em: <http://www.br-ie.org/pub/index.php/sbie/article/view/7998/5692>. Acesso em: Janeiro de 2019.

SCAICO, P. D. ; ALENCAR, Y. M.; et al. Relato da Utilização de uma Metodologia de Trabalho para o Ensino de Ciência da Computação no Ensino Médio. Anais do $18^{\circ}$ Workshop de Informática na Escola (WIE 2012) - Simpósio Brasileiro de Informática na Educação, 2012, Rio de Janeiro.

SBC. (2018). Diretrizes para ensino de Computação na Educação Básica. Disponível em: $\quad<$ http://www.sbc.org.br/educacao/diretrizes-para-ensino-de-computacao-naeducacao-basica $>$. Acesso em: Março de 2019.

Werlich, C.; Kemczinski, A.; Gasparini, I. (2018). "Pensamento Computacional no Ensino Fundamental: um mapeamento sistemático". Disponível em: $<$ http://www.tise.cl/Volumen14/TISE2018/375.pdf $>$. Acesso em: Janeiro de 2019.

Zanetti1, H. A. P.; Borges1, M. A. F.; Ricarte1, I. L. M. (2016). "Pensamento Computacional no Ensino de Programação: Uma Revisão Sistemática da Literatura Brasileira". Disponível em: $\quad<$ http://www.brie.org/pub/index.php/sbie/article/view/6677/4566>. Acesso em: Janeiro 2019.

Wing, J. M. (2006). Computational thinking.Commun. ACM, 49(3):33-35. 